\title{
新型 2-取代苯甲酰基-5-烷基环戊酮的设计、合成与生物活性研究
}

\author{
徐海珍 $*, a$ 朱有全 $*, b$ 谢丽芬 ${ }^{b}$ 王晓芳 $b$ \\ ${ }^{a}$ 天津师范大学化学学院 天津 300387) \\ ( ${ }^{b}$ 南开大学元素有机化学国家重点实验室 天津 300071)
}

\begin{abstract}
摘要 为了发现具有良好生物活性的新型先导化合物, 在综合多种高活性化合物结构特点的基础上, 设计合成了一系 列含苯甲酰基乙烯醇结构的 2-取代苯甲酰基-5-烷基-环戊酮类化合物. 以已二酸二乙酯为原料, 经 6 步反应制得目标产 物, 其结构经 IR, ${ }^{1} \mathrm{H}$ NMR, X 射线及元素分析或高分辨质谱确证, 并对该类化合物合成方法进行了探讨. 初步生测结果 表明，部分化合物对油菜表现出明显的抑制活性，在 $100 \mu \mathrm{g} / \mathrm{mL}$ 浓度下对油菜的抑制率达到 $60 \%$ 以上.
\end{abstract}

关键词 对羟基苯基丙酮酸双氧化酶; 芳酰基乙烯醇; 环戊酮; 除草活性

\section{Design, Synthesis and Bioactivity of Novel 2-Aroyl-5-alkylcyclopentanone Derivatives}

\author{
Xu, Haizhen ${ }^{*, a} \quad$ Zhu, Youquan ${ }^{*, b} \quad$ Xie, Lifen $^{b} \quad$ Wang, Xiaofang ${ }^{b}$ \\ $\left({ }^{a}\right.$ College of Chemistry, Tianjin Normal University, Tianjin 300387) \\ $\left({ }^{b}\right.$ State Key Laboratory of Elemento-organic Chemistry, Nankai University, Tianjin 300071)
}

\begin{abstract}
To find new lead compounds with muti-bioactivities, a series of novel 2-aroyl-5-alkylcyclopentanone derivatives containing 2-bezoylenthenol were designed on the base of various commercial products. The target compounds were synthesized from diethyl adipate in 6 steps. Their structures were confirmed by IR, ${ }^{1} \mathrm{H}$ NMR spectra, X-ray and elemental analysis or HRMS. The preliminary bioassay results indicated that some compounds showed obvious inhibition effects against Brassica campestris, and the herbicidal activities were more than $60 \%$ at $100 \mu \mathrm{g} / \mathrm{mL}$.

Keywords 4-hydroxyphenylpyruvate dioxygenase; 2-bezoylenthenol; cyclopropanone; herbicidal activity
\end{abstract}

对羟基苯基丙酮酸双氧化酶(简称 HPPD，EC 1.13.11.27)抑制剂是一类新的需光型除草剂 ${ }^{[1]}$, 许多已 商品化的该类化合物在结构上一般都具有芳酰基乙烯 醇结构(1)(图 1), 以保证它具有与对羟基苯基丙酩酸(简 称 HPP) 竞争 HPPD 活性中心 $\mathrm{Fe}^{2+}$ 进行络合的能力, 进而 导致作物茎叶白化死亡 ${ }^{[2]}$. 该类除草剂因其具有广谱的 除草活性，芽前和芽后均可使用的作用特点，使其衍生 物在农药中有着特殊的地位, 其衍生物的优异生物活性 更是吸引了许多农药化学家的注意, 不断有新型的衍生 物被研究和开发, 主要集中在三酮类(如 2-芳酰基环己 二酮类 2) 和二酮类(如 4-芳酰基吡唑类化合物 3 两类化 合物(图 1) ${ }^{[2,3]}$. 化合物 $\mathbf{2}$ 和 $\mathbf{3}$ 构效关系研究表明, 结构 $\mathbf{1}$ 是该类化合物的关键结构, 决定了该类化合物除草活
性，另外一个羰基为增效基团 ${ }^{[3,4]}$. 同时我们也注意到, 3-芳酰基吡咯烷-2,4-二酮类化合物 4 与 2 和 3 两类化合 物具有不同的构效关系: 前者为芳环上带有供电子基时 比拉电子基团的化合物活性高，后者相反 ${ }^{[5 \sim 9]}$. 与三酮 类化合物 $\mathbf{2}$ 相比, 二酮环缩小; 而与 $\mathbf{3}$ 相比, 环的大小相 同，但 3 类化合物的吡唑环具有芳香性，有利于羰基的 烯醇化，便于更大程度形成关键结构 $\mathbf{1}$, 而 4 类化合物 的吡咯环不具有芳香性，这可能是导致构效关系不同的 原因之一. 为进一步研究二/三酮类化合物的构效关系 和开发新型除草剂分子, 本工作结合 $\mathbf{2 , 3}$ 和 $\mathbf{4}$ 三类化合 物的结构特点，设计并采用下列路线合成了一系列含苯 甲酰基乙烯醇官能结构的 2-取代苯甲酰基-5-烷基-环戊 酮类化合物 9 (Scheme 1), 探讨该类杂环化合物的合成

\footnotetext{
*E-mail: hxxyxhz@mail.tjnu.edu.cn; zyq8165@nankai.edu.cn

Received August 28, 2012; revised September 22, 2012; published online September 26, 2012.

Project support by the National Basic Research Program of China (973 Program) (No. 2010CB126103), the National Natural Science Foundation of China (Nos. 20772066, 21072108) and the National Key Technologies R\&D Program (No. 2011BAE06B05-3).

国家 973 计划(No. 2010CB126103)、国家自然科学基金(Nos. 20772066, 21072108)和 “十二五” 科技支撑计划(No. 2011BAE06B05-3)资助项目.
} 
方法和生物活性.<smiles>O=C(O)c1ccccc1</smiles>

1<smiles>[R]c1cc(C(=O)c2c([R])nn([R])c2[R])c([R])c([R])c1[R]</smiles>

图 1 一些商品化品种的结构式

Figure 1 Structures of some commercial products
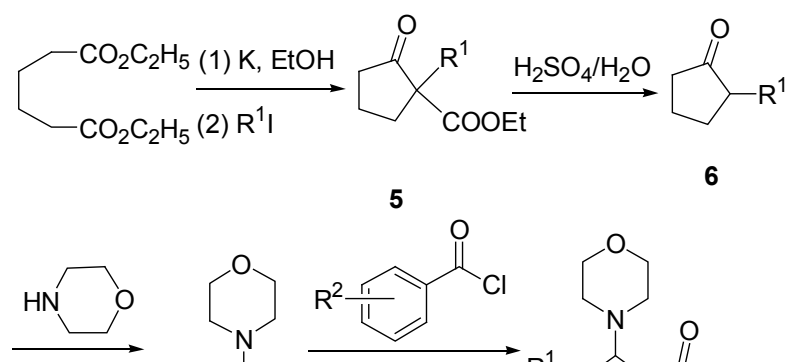<smiles>[R]C1CCC=C1N1CCOCC1</smiles><smiles>[R]c1ccc(C(=O)C2=C(N3CCOCC3)C([R])CC2)cc1</smiles><smiles></smiles>

9a $\mathrm{R}^{1}=\mathrm{CH}\left(\mathrm{CH}_{3}\right)_{2}, \mathrm{R}^{2}=\mathrm{H}$

9b $\mathrm{R}^{1}=\mathrm{CH}\left(\mathrm{CH}_{3}\right)_{2}, \mathrm{R}^{2}=4-\mathrm{Cl}$

9c $\mathrm{R}^{1}=\mathrm{CH}\left(\mathrm{CH}_{3}\right)_{2}, \mathrm{R}^{2}=2-\mathrm{CH}_{3}$

9d $\mathrm{R}^{1}=\mathrm{CH}\left(\mathrm{CH}_{3}\right)_{2}, \mathrm{R}^{2}=3-\mathrm{CH}_{3}$

9e $\mathrm{R}^{1}=\mathrm{CH}\left(\mathrm{CH}_{3}\right)_{2}, \mathrm{R}^{2}=4-\mathrm{CH}_{3}$

9f $\mathrm{R}^{1}=\mathrm{CH}\left(\mathrm{CH}_{3}\right)_{2}, \mathrm{R}^{2}=2,4-\mathrm{Cl}_{2}$

9g $\mathrm{R}^{1}=\mathrm{C}_{2} \mathrm{H}_{5}, \mathrm{R}^{2}=\mathrm{H}$

9h $\mathrm{R}^{1}=\mathrm{C}_{2} \mathrm{H}_{5}, \mathrm{R}^{2}=4-\mathrm{Cl}$

9i $\mathrm{R}^{1}=\mathrm{C}_{2} \mathrm{H}_{5}, \mathrm{R}^{2}=2-\mathrm{CH}_{3}$

9j $\mathrm{R}^{1}=\mathrm{C}_{2} \mathrm{H}_{5}, \mathrm{R}^{2}=4-\mathrm{CH}_{3}$

\section{Scheme 1}

\section{1 结果与讨论}

在设计合成 2-取代环戊酮(中间体 6)时, 我们首先 以环戊酮为原料, 通过以下反应路线制备(Scheme 2$)^{[10]}$.

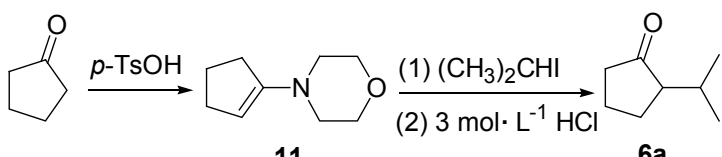

11
(2) $3 \mathrm{~mol} \cdot \mathrm{L}^{-1} \mathrm{HCl}$

6a

环戊酮与吗啡啉反应非常顺利，但是当反应产物 11 与碘代异丙烷反应时, 很难得到目标产物 6a. 这可能 是由于该反应属于 $\mathrm{S}_{\mathrm{N}} 2$ 亲核取代反应，中间体 11 的亲核 反应中心双键进攻异丙基碳时，异丙基形成的空间位阻 太大导致反应发生的几率大大降低, 进而得不到目标产 物 6. 为此, 我们采用以己二酸二乙酯为原料, 通过狄 克曼酯缩合得到 $\beta$-酩酸酯，再进行烷基化和酸化水解得 到中间体 $\mathbf{6}^{[11 \sim 13]}$, 此路线副产物少, 后处理简单, 简单 易行.

在目标产物的合成过程中，2-取代环戊酮在强碱条 件下夺取 $\alpha-\mathrm{H}$ 形成碳负离子再进行酰化也可能会得到 目标产物，但考虑到在强碱条件时，在相邻羰基的不同 位置都会发生酰化反应，也可能发生氧原子上的酰化反 应, 生成较多的副产物 ${ }^{[10]}$. 由于烯胺进行酰化时, 在相 邻羰基取代基较少的位置发生酰化反应，副产物少，因 此我们采用 2-取代环戊酮 6 与吗啡啉反应生成烯胺 7, 再进行酰化, 最后水解得到目标产物 $\mathbf{9}^{[13]}$. 在合成中间 体 $\mathbf{8}$ 时, 由于中间体 $\mathbf{7}$ 和酰氯都很活泼, 极易遇水分解, 所用溶剂乙醚和三乙胺都需要严格除水且反应体系需 在氮气保护下进行.

图谱解析：以化合物 $9 \mathrm{~g}$ 为例, $\delta \quad 0.89 \sim 1.02$ 处是甲 基氢, $\delta \quad 1.33 \sim 1.48$ 和 $1.51 \sim 1.64$ 处是乙基的 $\mathrm{CH}_{2}$ 两个 氢, $\delta 1.77 \sim 1.97,2.40 \sim 2.52,2.17 \sim 2.23$ 和 $2.77 \sim 2.82$ 处 是五元环上两个 $\mathrm{CH}_{2} \mathrm{CHEt}, \delta \quad 4.22 \sim 4.38$ 处是酮式结构 $\mathrm{COCHCO}$ 部分氢, $\delta 7.44 \sim 7.60$ 和 7.76 8.03 处是苯环 氢, $\delta 14.51$ 处是五元环 $\mathrm{CH}$ 烯醇后的吸收峰. 该化合物 分子中的两个羰基红外伸缩振动吸收峰位于 1737 和 $1674 \mathrm{~cm}^{-1}$.

根据我们前期的一些研究结果 ${ }^{[5-9]}$, 烯醇方式的不同 可能导致化合物构效关系的不同，尽管根据 Brown 等 ${ }^{[14]}$ 的研究结果, 酰基环戊酮的烯醇方式主要采取环外烯醇 方式，但考虑到本文所列化合物中含有多个位阻较大的 基团, 为了进一步了解目标化合物 9 采用环外还是环内 烯醇方式和指导今后的研究工作，在目标化合物均不适 合进行单晶结构测定的情况下, 我们将目标化合物 9a 进 一步衍生，与苯甲酰氯反应生成烯醇酯 $\mathbf{1 0}$ (Eq. 1)进行结 构测定, 单晶结构见图 $2^{[15]}$. 测定结果显示, 9a 与苯甲酰 氯形成了环外烯醇酯. 基于上述内容，我们认为该类化 合物的烯醇形式可能主要以环外形式为主.

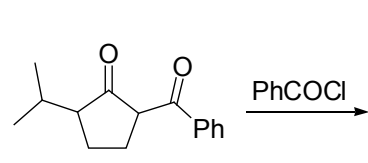

$9 a$

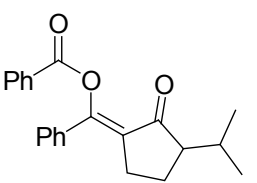

10

Scheme 2 


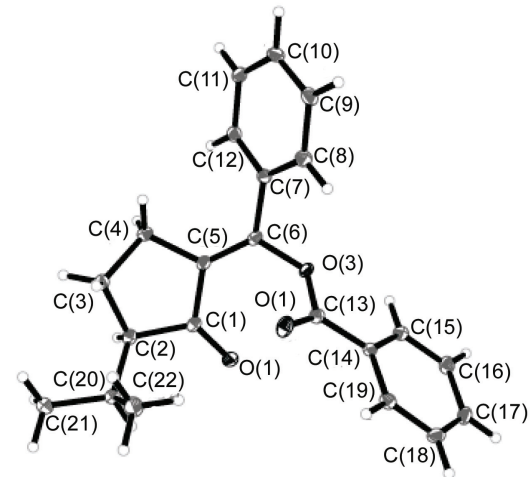

图 2 化合物 $\mathbf{1 0}$ 的晶胞结构图

Figure 2 The crystal structure of compound $\mathbf{1 0}$

构效关系讨论：目标化合物的除草活性见表 1. 田 间杂草的种类分为单子叶和双子叶两种杂草, 而油菜由 于属于敏感性的双子叶植物, 在测试化合物除草活性时 选择油菜作为双子叶杂草的代表, 而稗草(稗草为单子 叶杂草)为单子叶杂草的代表.

表 1 化合物 9a $~ 9 \mathbf{j}$ 的除草活性(抑制率/\%, 浓度 $100 \mu \mathrm{g} \cdot \mathrm{mL}^{-1}$ ) Table 1 Herbicidal activities of compounds $9 \mathbf{a} \sim \mathbf{9 j}$ (inhibiting rate $/ \%$, concentration: $100 \mu \mathrm{g} \bullet \mathrm{mL}^{-1}$ )

\begin{tabular}{ccc||ccc}
\hline Compd. & 油菜 & 稗草 & Compd & 油菜 & 稗草 \\
\hline 9a & 22.6 & 15.0 & $\mathbf{9 f}$ & 25.2 & 5.0 \\
$\mathbf{9 b}$ & 13.3 & 20.0 & $\mathbf{9 g}$ & 62.3 & 15.0 \\
$\mathbf{9 c}$ & 46.8 & 25.0 & $\mathbf{9 h}$ & 37.4 & 20.0 \\
$\mathbf{9 d}$ & 25.1 & 10.0 & $\mathbf{9 i}$ & 14.7 & 25.0 \\
$\mathbf{9 e}$ & 18.2 & 25.0 & $\mathbf{9 j}$ & 65.8 & 5.0 \\
\hline
\end{tabular}

从表 1 可以看出: 样品浓度为 $100 \mu \mathrm{g} / \mathrm{mL}$ 时, 化合 物对油菜表现出一定的抑制活性, 防除效果优于对稗草 的效果; $\mathrm{R}^{1}$ 为乙基时的抑制活性高于 $\mathrm{R}^{1}$ 为异丙基; $\mathrm{R}^{2}$ 为 供电子取代基时的除草活性高于 $\mathrm{R}^{2}$ 为吸电子取代基; 当 $R^{1}$ 为乙基, $R^{2}$ 分别为 $H(9 g)$ 和 $4-\mathrm{CH}_{3}(9 \mathrm{j})$ 时, 相应化合 物对油菜呈现 $60 \%$ 以上的抑制率. 而当光照条件下测试 对象为稗草时, 没有明显的规律可循.

\section{2 结论}

本工作为了发现具有良好生物活性的新型先导化 合物, 在综合多种高活性化合物结构特点的基础上, 设 计合成了一系列含苯甲酰基乙烯醇官能结构的 2-取代 苯甲酰氯-5-烷基-环戊酮类化合物, 并对该类化合物合 成方法和烯醇形式进行了探讨. 晶体结构分析表明, 环 外烯醇可能是目标产物的主要存在形式, 与之前我们报 道的吡咯烷酮类化合物的烯醇形式类似, 而不同于环己 二酮和吡唑类 HPPD 抑制剂分子. 初步生测结果表明, 部分化合物对油菜表现出明显的抑制活性, 在 100 $\mu \mathrm{g} / \mathrm{mL}$ 浓度下对油菜的抑制率达到 $60 \%$ 以上.

\section{3 实验部分}

\section{1 仪器与试剂}

北京科仪电光仪器厂XT 4A显微熔点测定仪, 温度 计未校正; Varain Mercury Plus $400 \mathrm{MHz}$ 核磁共振仪, TMS 为内标; Shimadzu-IR 435 红外光谱仪, 溴化钾压 片; Varian 7.0T FTICR-MS-ESI 高分辨质谱仪. Carlo Erba 1106 型元素分析仪. 实验用试剂和原料均为分析 纯或化学纯, 并根据需要进行了常规处理.

\section{2 实验方法}

3.2.1 目标化合物 2-取代苯甲酰基-5-烷基-环戊酮 9 的合成通法

参考文献[13]方法制备, 向 $50 \mathrm{~mL}$ 圆底烧瓶中加入 中间体 $8(5 \mathrm{mmol}), 3 \mathrm{~mol} \cdot \mathrm{L}^{-1}$ 稀盐酸 $(15 \mathrm{~mL})$ 和四氢呋喃 $(15 \mathrm{~mL})$, 加热搅拌回流 $5 \mathrm{~h}$, TLC 检测反应完全, 冷却, 静置分液, 分出有机层, 水层用二氯甲烷 $(10 \mathrm{~mL} \times 3)$ 萃 取, 合并有机层, 分别用饱和碳酸氢钠、饱和食盐水洗 涤, 用无水硫酸钠干燥. 抽滤, 真空减压旋出溶剂, 得 到粗产品, 柱层析得目标化合物 9.

2-苯甲酰基-5-异丙基环戍酮(9a): 淡黄色液体, 收 率 51\%. ${ }^{1} \mathrm{H} \mathrm{NMR}\left(\mathrm{CDCl}_{3}, 300 \mathrm{MHz}\right) \delta: 0.67 \sim 1.12[\mathrm{~m}$, $\left.6 \mathrm{H}, \mathrm{CH}\left(\mathrm{CH}_{3}\right)_{2}\right], 1.64 \sim 1.86(\mathrm{~m}, 1 \mathrm{H}, \mathrm{CHcycl}$ ), $1.96 \sim 2.12$ $\left[\mathrm{m}, 1 \mathrm{H}, \mathrm{CH}\left(\mathrm{CH}_{3}\right)_{2}\right], 2.15 \sim 2.98\left(\mathrm{~m}, 3 \mathrm{H}, \mathrm{CH}_{2} \mathrm{CH}_{2} \mathrm{CHcycl}\right.$ ), $2.40 \sim 2.61(\mathrm{~m}, 1 \mathrm{H}$, CHcycl. $), 4.08 \sim 4.46(\mathrm{~m}, 0.31 \mathrm{H}$, OCCHCO), $7.35 \sim 7.65(\mathrm{~m}, 3 \mathrm{H}, \mathrm{ArH}), 7.72 \sim 8.11(\mathrm{~m}, 2 \mathrm{H}$, ArH), 14.66 (br.s, 0.69H, OH); IR (KBr) v: 3439, 3006, $2960,1730 \mathrm{~cm}^{-1}$; HRMS calcd for $\mathrm{C}_{15} \mathrm{H}_{18} \mathrm{O}_{2} \mathrm{Na}[\mathrm{M}+\mathrm{Na}]^{+}$ 253.1199, found 253.1202.

2-(4-氯苯甲酰基)-5-异丙基环成酮(9b): 淡黄色固 体, 收率 54\%. m.p. 46 48 ${ }^{\circ} \mathrm{C} ;{ }^{1} \mathrm{H}$ NMR $\left(\mathrm{CDCl}_{3}, 300\right.$ $\mathrm{MHz}) \delta: 0.67 \sim 1.13\left[\mathrm{~m}, 6 \mathrm{H}, \mathrm{CH}\left(\mathrm{CH}_{3}\right)_{2}\right], 1.64 \sim 1.85(\mathrm{~m}$, $1 \mathrm{H}, \mathrm{CHcycl}.), 1.99 \sim 2.15\left[\mathrm{~m}, 1 \mathrm{H}, \mathrm{CH}\left(\mathrm{CH}_{3}\right)_{2}\right], 2.14 \sim 2.88$ (m, 3H, $\mathrm{CH}_{2} \mathrm{CH}_{2} \mathrm{CHcycl}$ ), $2.41 \sim 2.62$ (m, 1H, CHcycl.), $4.08 \sim 4.39(\mathrm{~m}, 0.27 \mathrm{H}, \mathrm{OCCHCO}), 7.36 \sim 7.54(\mathrm{~m}, 2 \mathrm{H}$, ArH), $7.68 \sim 8.16$ (m, 2H, ArH), 14.65 (br s, 0.73H, OH); IR (KBr) $v: 3453,2961,1736,1632 \mathrm{~cm}^{-1}$. Anal. calcd for $\mathrm{C}_{15} \mathrm{H}_{17} \mathrm{ClO}_{2}$ : C 68.05, H 6.47; found C 67.89, H 6.26.

2-(2-甲基苯甲酰基)-5-异丙基环戊醞(9c): 白色固 体, 收率 22\%. m.p. 44 46 ${ }^{\circ} \mathrm{C} ;{ }^{1} \mathrm{H}$ NMR $\left(\mathrm{CDCl}_{3}, 300\right.$ $\mathrm{MHz}) \delta: 0.83 \sim 1.11\left[\mathrm{~m}, 6 \mathrm{H}, \mathrm{CH}\left(\mathrm{CH}_{3}\right)_{2}\right], 1.63 \sim 1.78(\mathrm{~m}$, $1 \mathrm{H}, \mathrm{CHcycl}.), 1.91 \sim 2.06\left[\mathrm{~m}, 1 \mathrm{H}, \mathrm{CH}\left(\mathrm{CH}_{3}\right)_{2}\right], 2.15 \sim 2.57$ (m, 3H, $\mathrm{CH}_{2} \mathrm{CH}_{2} \mathrm{CHcycl}$ ), $2.34 \sim 2.46$ (m, 1H, CHcycl.), $2.41\left(\mathrm{~s}, 3 \mathrm{H}, \mathrm{CH}_{3}\right), 7.14 \sim 7.39(\mathrm{~m}, 4 \mathrm{H}, \mathrm{ArH}), 14.01$ (br s, $1 \mathrm{H}, \mathrm{OH})$; IR (KBr) v: 3439, 2959, $1737,1613 \mathrm{~cm}^{-1}$. HRMS calcd for $\mathrm{C}_{16} \mathrm{H}_{20} \mathrm{O}_{2} \mathrm{Na}[\mathrm{M}+\mathrm{Na}]^{+} 267.1355$, found 


\subsection{4 .}

2-(3-甲基苯甲酰基)-5-异丙基环戊酮(9d): 淡黄色 液体, 收率 40\%. ${ }^{1} \mathrm{H}$ NMR $\left(\mathrm{CDCl}_{3}, 300 \mathrm{MHz}\right) \delta: 0.74 \sim$ $1.11\left[\mathrm{~m}, 6 \mathrm{H}, \mathrm{CH}\left(\mathrm{CH}_{3}\right)_{2}\right], 1.61 \sim 1.78(\mathrm{~m}, 1 \mathrm{H}, \mathrm{CHcycl}$ ), $1.96 \sim 2.10\left[\mathrm{~m}, 1 \mathrm{H}, \mathrm{CH}\left(\mathrm{CH}_{3}\right)_{2}\right], 2.39$ (s, 3H, $\left.\mathrm{CH}_{3}\right), 2.11 \sim$ $2.82\left(\mathrm{~m}, 3 \mathrm{H}, \mathrm{CH}_{2} \mathrm{CH}_{2} \mathrm{CHcycl}\right.$ ), $2.44 \sim 2.55(\mathrm{~m}, 1 \mathrm{H}$, CHcycl.), $4.12 \sim 4.42(\mathrm{~m}, 0.17 \mathrm{H}, \mathrm{OCCHCO}), 7.17 \sim 7.44$ (m, 2H, ArH), 7.49 7.92 (m, 2H, ArH), 14.69 (br s, $0.83 \mathrm{H}, \mathrm{OH})$; IR (KBr) $v: 3439,2959,1737,1613 \mathrm{~cm}^{-1}$; HRMS calcd for $\mathrm{C}_{16} \mathrm{H}_{20} \mathrm{O}_{2} \mathrm{Na}[\mathrm{M}+\mathrm{Na}]^{+}$267.1355, found 267.1353.

2-(4-甲基苯甲酰基)-5-异丙基环戊酮(9e)：淡黄色 液体，收率 $16 \% .{ }^{1} \mathrm{H}$ NMR $\left(\mathrm{CDCl}_{3}, 300 \mathrm{MHz}\right) \delta: 0.70 \sim$ $1.15\left[\mathrm{~m}, 6 \mathrm{H}, \mathrm{CH}\left(\mathrm{CH}_{3}\right)_{2}\right], 1.62 \sim 1.80(\mathrm{~m}, 1 \mathrm{H}, \mathrm{CHcycl}$ ), $1.95 \sim 2.12\left[\mathrm{~m}, 1 \mathrm{H}, \mathrm{CH}\left(\mathrm{CH}_{3}\right)_{2}\right], 2.40\left(\mathrm{~s}, 3 \mathrm{H}, \mathrm{CH}_{3}\right), 2.12 \sim$ $2.87\left(\mathrm{~m}, 3 \mathrm{H}, \mathrm{CH}_{2} \mathrm{CH}_{2} \mathrm{CHcycl}\right.$ ), $2.43 \sim 2.60(\mathrm{~m}, 1 \mathrm{H}$, CHcycl.), $4.09 \sim 4.41(\mathrm{~m}, 0.39 \mathrm{H}, \mathrm{OCCHCO}), 7.14 \sim 7.36$ $(\mathrm{m}, 2 \mathrm{H}, \mathrm{ArH}), 7.64 \sim 8.13(\mathrm{~m}, 2 \mathrm{H}, \mathrm{ArH}), 14.75$ (br s, 0.63H, OH); IR (KBr) v: 3452, 2959, 2872, 1736, 1609 $\mathrm{cm}^{-1}$; HRMS calcd for $\mathrm{C}_{16} \mathrm{H}_{20} \mathrm{O}_{2} \mathrm{Na}[\mathrm{M}+\mathrm{Na}]^{+}$267.1355, found 267.1357 .

2-(2,4-二氯苯甲酰基)-5-异丙基环戊酮(9f): 淡黄色 液体，收率 $72 \% .{ }^{1} \mathrm{H}$ NMR $\left(\mathrm{CDCl}_{3}, 300 \mathrm{MHz}\right) \delta: 0.77 \sim$ $1.12\left[\mathrm{~m}, 6 \mathrm{H}, \mathrm{CH}\left(\mathrm{CH}_{3}\right)_{2}\right], 1.62 \sim 1.77(\mathrm{~m}, 1 \mathrm{H}, \mathrm{CHcycl}$ ), $1.94 \sim 2.04\left[\mathrm{~m}, 1 \mathrm{H}, \mathrm{CH}\left(\mathrm{CH}_{3}\right)_{2}\right], 2.15 \sim 2.26(\mathrm{~m}, 1 \mathrm{H}$, CHcycl.), $2.27 \sim 2.35$ (m, 1H, CHcycl.), 2.35 2.44 (m, $1 \mathrm{H}$, CHcycl.), $2.44 \sim 2.57$ (m, 1H, CHcycl.), 7.28 7.39 (m, 2H, ArH), $7.43 \sim 7.51$ (m, 1H, ArH), 13.67 (br s, 1H, $\mathrm{OH})$; IR (KBr) $v: 3452,2965,1702,1586 \mathrm{~cm}^{-1}$. Anal. calcd for $\mathrm{C}_{15} \mathrm{H}_{16} \mathrm{Cl}_{2} \mathrm{O}_{2}$ : C 60.22 , H 5.39; found $\mathrm{C} 60.43, \mathrm{H}$ 5.36 .

2-苯甲酰基-5-乙基环戊酮 $(9 \mathrm{~g})$ ：淡黄色液体，收率 $20 \% .{ }^{1} \mathrm{H}$ NMR $\left(\mathrm{CDCl}_{3}, 300 \mathrm{MHz}\right) \delta: 0.89 \sim 1.02(\mathrm{~m}, 3 \mathrm{H}$, $\left.\mathrm{CH}_{2} \mathrm{CH}_{3}\right), 1.33 \sim 1.48\left(\mathrm{~m}, 1 \mathrm{H}, \mathrm{CH}_{2} \mathrm{CH}_{3}\right), 1.51 \sim 1.64(\mathrm{~m}$, $1 \mathrm{H}, \mathrm{CH}_{2} \mathrm{CH}_{3}$ ), $1.77 \sim 1.97$ (m, 1H, CHcycl.), $2.17 \sim 2.82$ (m, 3H, $\mathrm{CH}_{2} \mathrm{CH}_{2} \mathrm{CHcycl}$.), $2.40 \sim 2.52$ (m, 1H, CHcycl.), $4.22 \sim 4.38(\mathrm{~m}, 0.28 \mathrm{H}, \mathrm{OCCHCO}), 7.44 \sim 7.60(\mathrm{~m}, 3 \mathrm{H}$, $\mathrm{ArH}), 7.76 \sim 8.03(\mathrm{~m}, 2 \mathrm{H}, \mathrm{ArH}), 14.51$ (br s, $0.72 \mathrm{H}, \mathrm{OH})$; IR (KBr) v: 3453, 2962, 2873, 1737, $1674 \mathrm{~cm}^{-1}$; HRMS calcd for $\mathrm{C}_{14} \mathrm{H}_{16} \mathrm{O}_{2} \mathrm{Na}[\mathrm{M}+\mathrm{Na}]^{+}$239.1043, found 239.1048.

2-(4-氯苯甲酰基)-5-乙基环戊酮(9h)：淡黄色固体, 收率 41\%. m.p. 52 54 ${ }^{\circ} \mathrm{C} ;{ }^{1} \mathrm{H}$ NMR $\left(\mathrm{CDCl}_{3}, 300 \mathrm{MHz}\right)$ $\delta: 1.02\left(\mathrm{t}, J=7.5 \mathrm{~Hz}, 3 \mathrm{H}, \mathrm{CH}_{2} \mathrm{CH}_{3}\right), 1.36 \sim 1.52(\mathrm{~m}, 1 \mathrm{H}$, $\left.\mathrm{CH}_{2} \mathrm{CH}_{3}\right), 1.53 \sim 1.70\left(\mathrm{~m}, 1 \mathrm{H}, \mathrm{CH}_{2} \mathrm{CH}_{3}\right), 1.85 \sim 2.08(\mathrm{~m}$,
1H, CHcycl.), 2.16 2.33 (m, 1H, CHcycl.), 2.40 2.54 (m, 1H, CHcycl.), 2.70 2.87 (m, 2H, $\mathrm{CH}_{2}$ cycl.), 7.44 (d, $J=8.6 \mathrm{~Hz}, 2 \mathrm{H}, \mathrm{ArH}), 7.73$ (d, $J=8.6 \mathrm{~Hz}, 2 \mathrm{H}, \mathrm{ArH}), 14.50$ (br s, 1H, OH); IR (KBr) v: 3446, 2962, 2873, 1737, 1675 $\mathrm{cm}^{-1}$. Anal. calcd for $\mathrm{C}_{14} \mathrm{H}_{15} \mathrm{ClO}_{2}$ : C 67.07, H 6.03; found C 67.09, H 5.91.

2-(2-甲基苯甲酰基)-5-乙基环戊酮(9i): 淡黄色液 体，收率 $25 \% .{ }^{1} \mathrm{H} \mathrm{NMR}\left(\mathrm{CDCl}_{3}, 300 \mathrm{MHz}\right) \delta: 0.82 \sim 1.08$ $\left(\mathrm{m}, 3 \mathrm{H}, \mathrm{CH}_{2} \mathrm{CH}_{3}\right), 1.34 \sim 1.46\left(\mathrm{~m}, 1 \mathrm{H}, \mathrm{CH}_{2} \mathrm{CH}_{3}\right), 1.48 \sim$ $1.63\left(\mathrm{~m}, 1 \mathrm{H}, \mathrm{CH}_{2} \mathrm{CH}_{3}\right), 1.80 \sim 2.00(\mathrm{~m}, 1 \mathrm{H}, \mathrm{CHcycl}$ ), $2.07 \sim 2.23$ (m, 1H, CHcycl.), 2.39 (s, 3H, $\left.\mathrm{CH}_{3}\right), 2.29 \sim$ 2.56 (m, 3H, $\mathrm{CH}_{2} \mathrm{CHcycl}$ ), $4.07 \sim 4.39$ (m, 0.20H, OCCHCO), $7.13 \sim 7.85$ (m, 4H, ArH), 13.84 (br s, $0.80 \mathrm{H}$, $\mathrm{OH})$; IR (KBr) v: 3451, 3006, 2962, 1720, $1593 \mathrm{~cm}^{-1}$; HRMS calcd for $\mathrm{C}_{15} \mathrm{H}_{18} \mathrm{O}_{2} \mathrm{Na}[\mathrm{M}+\mathrm{Na}]^{+}$253.1199, found 253.1202.

2-(4-甲基苯甲酰基)-5-乙基环戊酮(9j): 淡黄色液 体，收率 31\%. ${ }^{1} \mathrm{H}$ NMR $\left(\mathrm{CDCl}_{3}, 300 \mathrm{MHz}\right) \delta: 0.67 \sim 0.98$ (m, $\left.3 \mathrm{H}, \mathrm{CH}_{2} \mathrm{CH}_{3}\right), 1.19 \sim 1.35\left(\mathrm{~m}, 1 \mathrm{H}, \mathrm{CH}_{2} \mathrm{CH}_{3}\right), 1.37 \sim$ $1.57\left(\mathrm{~m}, 1 \mathrm{H}, \mathrm{CH}_{2} \mathrm{CH}_{3}\right), 1.65 \sim 1.89$ (m, 1H, CHcycl.), 2.29 (s, 3H, $\left.\mathrm{CH}_{3}\right), 1.98 \sim 2.84\left(\mathrm{~m}, 3 \mathrm{H}, \mathrm{CH}_{2} \mathrm{CH}_{2} \mathrm{CHcycl}\right.$ ), $2.20 \sim 2.51(\mathrm{~m}, 1 \mathrm{H}, \mathrm{CHcycl}),. 4.03 \sim 4.33(\mathrm{~m}, 0.38 \mathrm{H}$, OCCHCO), 7.15 (t, $J=8.4 \mathrm{~Hz}, 2 \mathrm{H}, \mathrm{ArH}), 7.50 \sim 7.91(\mathrm{~m}$, $2 \mathrm{H}, \mathrm{ArH}), 14.48$ (br s, 0.62H, OH); IR (KBr) v: 3446, 2961, 2929, 1737, $1671 \mathrm{~cm}^{-1}$; HRMS calcd for $\mathrm{C}_{15} \mathrm{H}_{18} \mathrm{O}_{2} \mathrm{Na}[\mathrm{M}+\mathrm{Na}]^{+}$253.1199, found 253.1203

3.2.2（Z)-(3-异丙基-2-氧代环戊亚基)(苯基)甲基苯 甲酸酯(10)的制备

氮气保护下, 向 $25 \mathrm{~mL}$ 两口烧瓶中加入 $0.23 \mathrm{~g} \mathrm{(1}$ $\mathrm{mmol}$ ) 2-苯甲酰基-5-异丙基环戊酮(9a)，0.11 g (1.1 $\mathrm{mmol}$ )三乙胺和 $2 \mathrm{~mL}$ THF, 再慢慢滴加入 $0.14 \mathrm{~g}$ (1 $\mathrm{mmol}$ )苯甲酰氯和 $2 \mathrm{~mL} \mathrm{THF}$, 滴加过程中反应液变浑 浊, 滴加完毕后，室温摚拌 $1 \mathrm{~h}, \mathrm{TLC}$ 检测反应完全，加 入 $5 \mathrm{~mL}$ 水溶解铵盐, 用乙酸乙酯 $(5 \mathrm{~mL} \times 3)$ 萃取, 合并 有机层, 用无水硫酸钠干燥. 抽滤, 真空减压旋出溶剂,

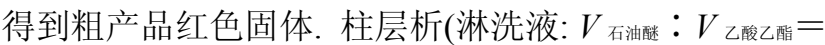
$4: 1$ ) 得到 $0.27 \mathrm{~g}$ 无色晶体 10, 收率 $81 \%$. m.p. 108 110 ${ }^{\circ} \mathrm{C} ;{ }^{1} \mathrm{H}$ NMR $\left(\mathrm{CDCl}_{3}, 300 \mathrm{MHz}\right) \delta: 0.77 \sim 1.05[\mathrm{~m}, 6 \mathrm{H}$, $\left.\mathrm{CH}\left(\mathrm{CH}_{3}\right)_{2}\right], 1.53 \sim 1.79(\mathrm{~m}, 1 \mathrm{H}, \mathrm{CHcycl}$ ), $2.00 \sim 2.14[\mathrm{~m}$, $\left.1 \mathrm{H}, \mathrm{CH}\left(\mathrm{CH}_{3}\right)_{2}\right], 1.81 \sim 2.34$ (m, $2 \mathrm{H}, \mathrm{CH}_{2} \mathrm{CH}_{2} \mathrm{CHcycl}$ ), $2.75 \sim 2.99\left(\mathrm{~m}, 2 \mathrm{H}, \mathrm{CH}_{2} \mathrm{CH}_{2} \mathrm{CHcycl}\right.$ ), $7.23 \sim 7.33(\mathrm{~m}, 1 \mathrm{H}$, ArH), $7.40 \sim 7.42(\mathrm{~m}, 2 \mathrm{H}, \mathrm{ArH}), 7.46 \sim 7.51(\mathrm{~m}, 2 \mathrm{H}, \mathrm{ArH})$, $7.58 \sim 7.72(\mathrm{~m}, 3 \mathrm{H}, \mathrm{ArH}), 8.19(\mathrm{~d}, J=7.7 \mathrm{~Hz}, 2 \mathrm{H}, \mathrm{ArH})$.

\subsection{3 化合物 $9 \mathbf{a} \sim 9 \mathbf{j}$ 的生测方法}

油菜平血法: 直径 $6 \mathrm{~cm}$ 的培养皿中铺好一张直径 
$5.6 \mathrm{~cm}$ 的滤纸, 加入 $2 \mathrm{~mL} 100 \mu \mathrm{g} \cdot \mathrm{mL}^{-1}$ 的供试化合物溶 液, 播种浸种 $4 \sim 6 \mathrm{~h}$ 的油菜种子 15 粒, $(28 \pm 1){ }^{\circ} \mathrm{C}$ 下, 黑 暗培养 $66 \mathrm{~h}$ 后测定胚根长度. 通过黑暗条件下化合物对 油菜胚根的生长抑制来检测化合物的除草活性 ${ }^{[5,9,16 ~ 19]}$.

稗草小杯法: 通过光照条件下对稗草幼苗株高的生 长抑制来检测化合物的除草活性. 测试浓度为 $100 \mu \mathrm{g}$ ・ $\mathrm{mL}^{-1}$. 活性指标为株高生长抑制率 $(\%)^{[5,9,16 \sim 19]}$.

辅助材料(Supporting Information) 辅助材料为化合 物 $5,6,7$ 及 8 的制备及化合物 10 的晶体结构数据. 这些 材料可以免费从本刊网站(http://sioc-journal.cn/)上下载.

\section{References}

[1] Wu, Y. C.; Hu, F. Z.; Yang, H. Z. Chin. J. Pestic. Sci. 2001, 3,1 (in Chinese).

(吴彦超, 胡方中, 杨华铮, 农药学学报, 2001, 3, 1.)

[2] Zhu, Y. Q.; Hu, F. Z.; Yang, H. Z.; Xu, H. Z. Chemistry 2004, 67, w018 (in Chinese).

(朱有全, 胡方中, 杨华铮, 徐海珍, 化学通报, 2004, 67, w018.)

[3] Lee, D. L.; Prisbylla, M. P.; Cromartie, T. H.; Dagarin, D. P.; Howard, S. W.; Provan, W. M.; Ellis, M. K.; Fraser, T.; Mutter, L. C. Weed Sci. 1997, 45, 601.

[4] Wu, C.-S.; Huang, J.-L.; Sun Y.-S.; Yang, D.-Y. J. Med. Chem. 2002, 45, 2222.

[5] Zhu, Y. Q.; Si, X. K.; Zou, X. M.; Liu, B.; Yang, H. Z. Chin. J. Org. Chem. 2007, 27, 385 (in Chinese).

(朱有全, 司学凯, 邹小毛, 刘斌, 杨华铮, 有机化学, 2007, 27, 385.)

[6] Zhu, Y. Q.; Zou, X. M.; Hu, F. Z.; Yao, C. S.; Liu, B.; Yang, H. Z. J. Agric. Food Chem. 2005, 53, 9566.

[7] Zhu, Y. Q.; Liu, P.; Si, X. K.; Zou, X. M.; Liu, B.; Song, H. B.;
Yang, H. Z. J. Agric. Food Chem. 2006, 54, 7200.

[8] Zhu, Y. Q.; Yao, C. S.; Zou, X. M.; Hu, F. Z.; Liu, B.; Yang, H. Z. Molecules 2005, 10, 427.

[9] Zhu, Y. Q.; Hu, F. Z.; Zou, X. M.; Yao, C. S.; Liu, B.; Li, Y. H.; Yang, H. Z. Chin. J. Org. Chem. 2005, 25, 419 (in Chinese).

(朱有全, 胡方中, 邹小毛, 姚昌盛, 刘斌, 李永红, 杨华铮, 有 机化学, 2005, 25, 419.)

[10] Stork, G.; Brizzolara, A.; Landesman, H. J. Am. Chem. Soc. 1963, $85,207$.

[11] Magnus, P.; Shen, L. Tetrahedron 1999, 55, 3553.

[12] Francis, H.; Case, E.; Emmbt, R. J. Am. Chem. Soc. 1928, 50, 3062.

[13] Campbell, R. D.; Jung, J. A. J. Org. Chem. 1965, 30, 3711.

[14] Brown, H. C.; Brewster, J. H.; Shechter, H. J. Am. Chem. Soc. 1954, 76, 467.

[15] The crystal structure has been deposited at the Cambridge Crystallographic Data Centre and allocated the deposition number: CCDC 874907.

[16] Zhu, Y.-Q.; Liu, C.; Zhu, R.; Zhang, J.; Yuan, Y.-W.; Zou, X.-M.; Hu, F.-Z.; Yang, H.-Z. Chin. J. Org. Chem. 2010, 30, 1088 (in Chinese).

(朱有全, 刘翠, 朱然, 张金, 袁燕伟, 邹小毛, 胡方中, 杨华铮, 有机化学, 2010, 30, 1088.)

[17] Zhu, Y.-Q.; Zhu, R.; Yuan, Y.-W.; Zhang, J.; Zou, X.-M.; Hu, F.-Z.; Yang, H.-Z. Chin. J. Org. Chem. 2010, 30, 1207 (in Chinese).

(朱有全, 朱然, 袁燕伟, 张金, 邹小毛, 胡方中, 杨华铮, 有机 化学, 2010, 30, 1207.)

[18] Zhu, Y.-Q.; Liu, W.-M.; Liu, B.; Hu, F.-Z.; Zhu, R.; Zou, X.-M.; Yang, H.-Z. Chin. J. Org. Chem. 2009, 29, 638 (in Chinese). (朱有全, 刘卫敏, 刘斌, 胡方中, 朱然, 邹小毛, 杨华铮, 有机 化学, 2009, 29, 638.)

[19] Zhu, Y.-Q.; Wang, D.-Y.; Yuan, Y.-W.; Ma, Y.; Zou, X.-M.; Yang, H.-Z. Chin. J. Org. Chem. 2012, 32, 2115 (in Chinese). (朱有全, 王丹阳, 袁燕伟, 马源, 邹小毛, 杨华铮, 有机化学, 2012, 32, 2115.) 